\title{
Spatial Spectroscopy Approach for Detection of Internal Defect of Component without Zero-Position Sensors
}

\author{
Qizhou Wu, Yong Jin, Zhaoba Wang, and Zhaoqian Xiao \\ National Key Lab for Electronic Measurement Technology, North University of China, Taiyuan, Shanxi 030051, China \\ Correspondence should be addressed to Qizhou Wu; wqzpaper@126.com
}

Received 20 January 2016; Accepted 9 March 2016

Academic Editor: Yu Shang

Copyright (C) 2016 Qizhou Wu et al. This is an open access article distributed under the Creative Commons Attribution License, which permits unrestricted use, distribution, and reproduction in any medium, provided the original work is properly cited.

\begin{abstract}
Conventional approach to detect the internal defect of a component needs sensors to mark the "zero" positions, which is timeconsuming and lowers down the detecting efficiency. In this study, we proposed a novelty approach that uses spatial spectroscopy to detect internal defect of objects without zero-position sensors. Specifically, the spatial variation wave of distance between the detecting source and object surface is analyzed, from which a periodical cycle is determined with the correlative approaches. Additionally, a wavelet method is adopted to reduce the noise of the periodic distance signal. This approach is validated by the ultrasound detection of a component with round cross section and elliptical shape in axis. The experimental results demonstrate that this approach greatly saves the time spent on the judgment of a complete cycle and improves the detecting efficiency of internal defect in the component. The approach can be expanded to other physical methods for noninvasive detection of internal defect, such as optical spectroscopy or X-ray scanning, and it can be used for hybrid medium, such as biological tissues.
\end{abstract}

\section{Introduction}

The components with large change in the surface shape are often found in both industrial and biological fields. Ultrasonic detection of the internal defects in such components is of importance and becoming a hot topic among researchers [1]. In the process of detection, the ultrasonic probe is required to be kept in an invariable and certain distance from the surface of the component. Moreover, the incident angle of the probe should be kept perpendicular to the surface of the component. Traditionally, the component needs a whole scanning in a manner of constant speed and across multisections. In each scanning cycle, the triggering signals of "zero" position are detected by a sensor and taken as the beginning and ending signals of scanning. Then, the scanning on the whole component is made with fixed intervals. Once the scanning in each cycle is completed, the probe is moved at a fixed distance along the axial direction and waits again to receive the triggering signals from the "zero-" position sensor. The time spent for waiting generally is half of that spent in the scanning in one cycle. Therefore, the efficiency of the detection is very low.
In this paper, we proposed a novelty approach with correlative theory, called spatial spectroscopy, to continuously scan the component without waiting for triggering signals from "zero-" position sensor. Here the "spatial spectroscopy" indicates that the periodical signals of ultrasound backscatter, with the spatial change of the component, are used to detect the internal detects.

Additionally, we combined this approach with selfadaptive filter technology for improvement of the detection efficiency.

The self-adaptive filter is a signal processing technology that was originated since 1960s, and many researchers have contributed to this technology. For example, Xu et al. applied self-adaptive filter to the field of Internet engineering [2]. Chen et al. combined self-adaptive filter with neural network for use in image processing [3]. Feng and Lin utilized the self-adaptive filter to enhance the signal intensity and elevate the signal-to-noise ratio for A/D conversion [4]. Zhong et al. utilized self-adaptive filter to improve the searching engine in GPS (Global Positioning System) [5]. Recently, self-adaptive filter was introduced into ultrasonic detection of internal defects in components, with purpose of improving 


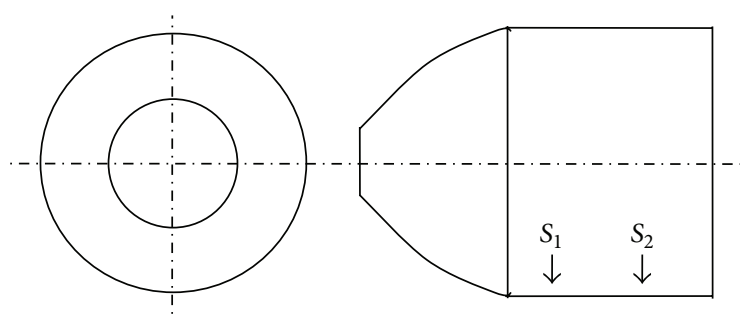

FIGURE 1: Structure of the component with round cross section and elliptical shape in axis.

the signal-to-noise ratio. The aim of using self-adaptive filter in this study is to smooth the ultrasonic signals before using spatial spectroscopy approach.

The correlative theory is more often utilized for signal matching. For example, Ji et al. and Ge et al. proposed methods that utilized correlative theory to extract the true signals from the data with weak signal-to-noise ratio $[6$, 7]. Wang and Ruan proposed an image-matching algorithm based on correlative theory [8]. Moreover, they proposed a pyramid-layered model to reduce the calculation loads due to correlation of images and improve the precision of image-matching. Lei et al. proposed a recognition method for internal defects based on correlative theory, which has become a standard for online or offline detection of internal defects during structure fluctuation [9].

To overcome the low detection efficiency of "zero-" position sensor method, we realized the fast localization of "zero" position by using the feature of high correlation in periodic signals. Through preprocessing by the self-adaptive filter, the ultrasonic signals exhibit excellent correlations, thus allowing for fast scanning of the component without "zero" sensor.

\section{Methods}

2.1. Analysis on the Inspection and Measurement on the Component. The component structure and defect positions $\left(S_{1}\right.$ and $S_{2}$ ) are shown in Figure 1. It has round cross section and elliptical shape in axis, $50 \mathrm{~mm}$ length and $101 \mathrm{~mm}$ diameter. The length and width of the defect are $18 \mathrm{~mm}$ and $1 \mathrm{~mm}$, respectively. The distance between the two defect positions is $29 \mathrm{~mm}$. The ultrasound backscatter signals received from the surface of component are depicted in Figure 2.

The approach for scanning without zero-position sensors is illustrated as follows. First, the scanning on the component with constant speed (3.14 rad/s in counterclockwise) started immediately after the ultrasonic probe is moved to the cross sections of the component, and the position of the zero point in each detection cross section can be any point in the cross section. When scanning on a certain component with an elongated defect without zero sensors the same defect can be detected in different angles on different cross sections, which directly affects the judgment on the defect, as depicted in Figure 3.

Because the detection target is a blank component, the time for each cycle is fixed when the component is being

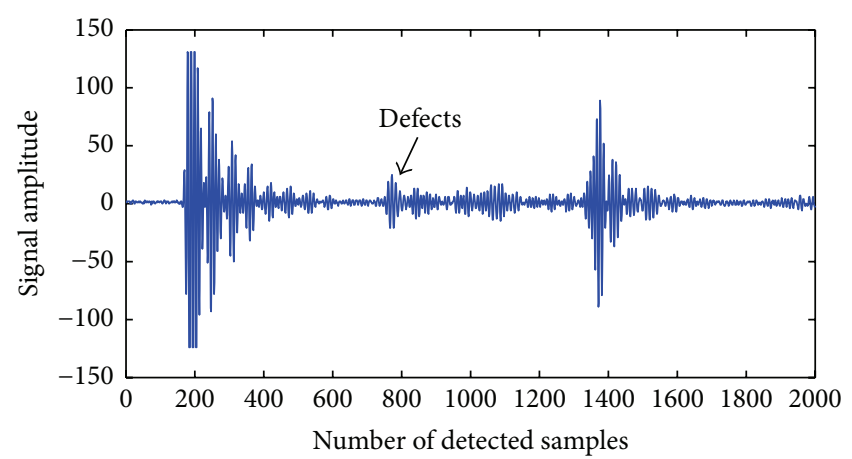

FIGURE 2: The ultrasound backscatter signals received from the surface of component.

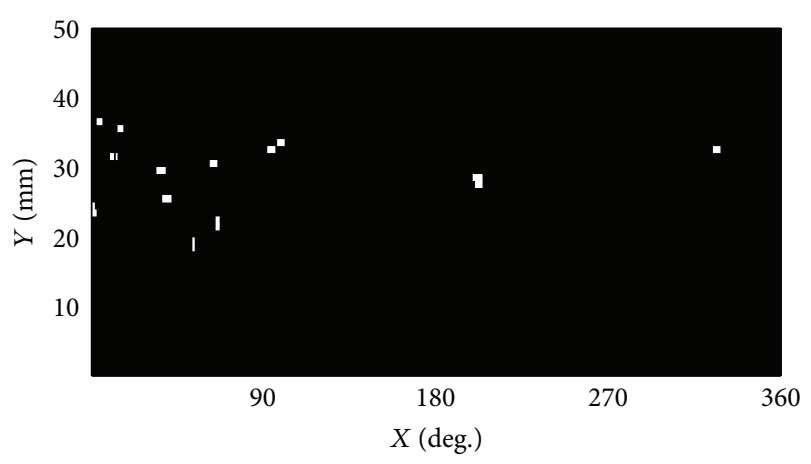

FIgURE 3: Results of the whole scanning on the component.

rotated with constant speed, and within this cycle the changes of the distance between the probe and the surface of the component can be detected, as shown in Figure 4.

After the scanning (rotate), the periodic signals in each detection cross section are extracted, covering the range of distance between the probes over the cross sections and the surface of the component. The cycles covering any cross sections are the same, if taking the periodic zero point in the first cross section as the circumference zero point. Based on this characteristic, a correlative judgment on the periodic zero points in all the cross sections and the zero point in the first detection cross section is made through the method of signal process, and matching zeros is implemented to make a rectification on the detection result.

2.2. Analyses on the Periodic Signal. The curve in Figure 4 represents the distance signal of 3 rotating cycles in a certain detected cross section, which is obtained with measurement of the distance between the probe and the surface of a certain component.

Affected by the on-engine high-frequency vibration resulting from the motor drive and other factors, there exists a kind of high-frequency noise in the periodic signal. Moreover, there may be some miniature saddle-backing and subsiding because the detected object is the blank component; in order to assure the similarity of the periodic signal in each cross section, the periodic signal obtained at the position of those saddle-backing and subsiding should be processed 


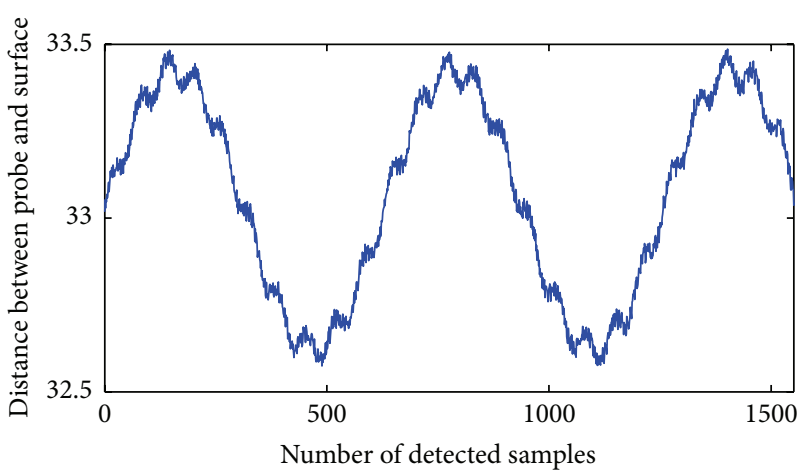

Figure 4: The relationship between the distance (from probe to surface, $\mathrm{mm}$ ) and number of the detected samples.

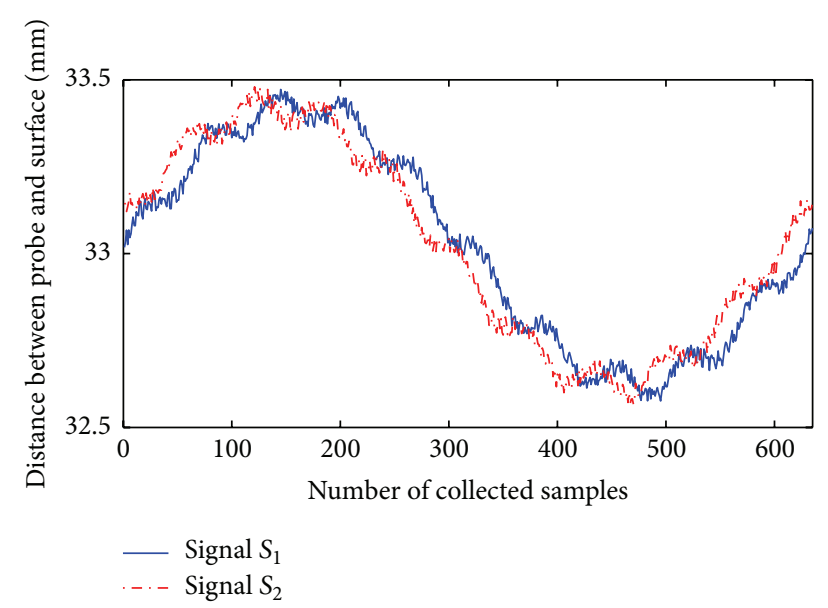

Figure 5: Distance $(\mathrm{mm})$ signals $S_{1}, S_{2}$ on the scanning cross sections.

as the frequency noise. Therefore, the noise involved in the signals should be reduced before matching zeros, so as to extract the periodic signals. Figure 5 is the sketch map for the distance signals $S_{1}$ and $S_{2}$ of two different cross sections obtained in the scanning on a certain component.

2.3. Reduction of the Noise Involved in Signals. During the scanning, the signal of distance between the probe and the surface of the component is treated as stable low-frequency signal, and the high-frequency part in the signal is the noise. As such, the influence of the high-frequency part in the signal should be eliminated before signal analysis is performed. Because the noise with different frequency components needs to be filtered out, a self-adapting noise reduction method with multilevel and multiresolution should be used [10-14]. Here wavelet analysis is used for this purpose [15]. The following are steps of the method for reducing the noise involved in signals.

(1) Select a wavelet and analyze the number of decomposition. Wavelet decomposition is made on the signals involving noise to obtain the low-frequency and highfrequency coefficients.



FIGURE 6: Distance signals $S_{1}$ and $S_{2}$ after the noise reduction.

(2) A suitable method is adopted to make an analysis on all the high-frequency coefficients, so as to remove the noise with frequency components.

(3) According to the levels number of decomposition on the wavelet, a reconstruction of the discrete wavelet is made on the processed high-frequency and lowfrequency coefficients. The acquired signals are those with noise reduction.

In addition, according to the selective analysis on the wavelet base and the levels number of decomposition on wavelet described in the reference, the wavelet $\mathrm{db} 5$ is used to make decomposition with 5 levels on the distance signals $S_{1}$ and $S_{2}$.

Furthermore, the method of HeurSure threshold value is adopted to make a soft threshold selection on the highfrequency coefficients in the different levels and filter out the noise with high-frequency, and a wavelet reconstruction is made on the filtered constituents to get the distance signals $S_{1}$ and $S_{2}$ after noise reduction with the method of multiresolution analysis, as indicated in Figure 6.

2.4. Matching Zeros. In the process of scanning, the signals obtained after the noise reduction on the collected distance signals are the distance signals in one cycle. In other words, the detected values are the measured distance in a single-cycle within one cross section of the component. Therefore, the inertial point in the cycle needs to be found so as to perform the correlative judgment on the zero points in the detected component and realize the matching zeros.

The mutually correlative function of the stochastic signals is used to describe the interdependent relationship between the two sample functions at the different time [16-19]. This function is used to describe the close mutual interdependent relationship between the waveforms of the two stochastic signals with the movement of the time coordinates. 


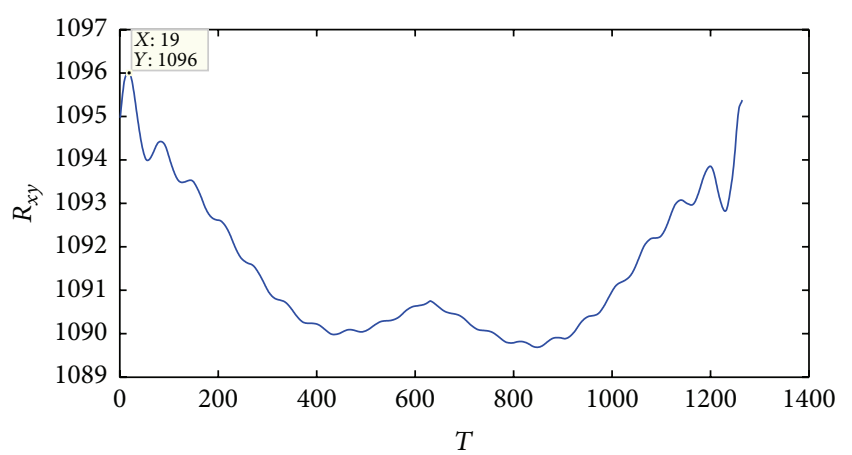

FIGURE 7: Cross correlative function $R_{x y}$ of $S_{1}$ and $S_{2}$.

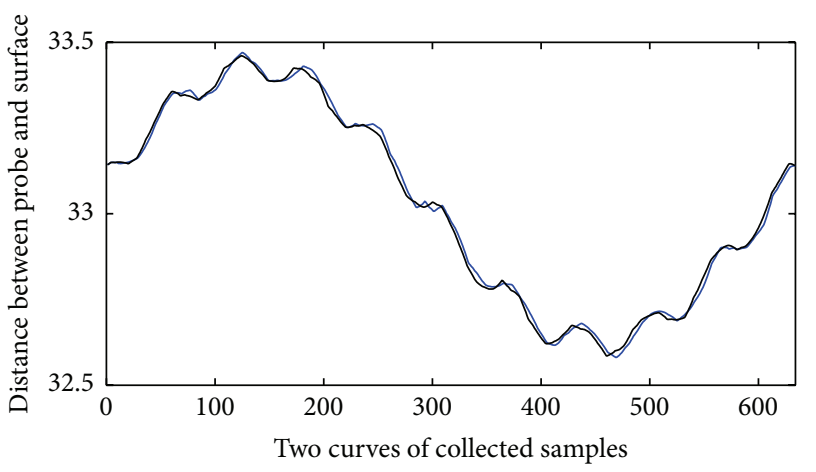

Figure 8: Comparison between $S_{1}$ (blue) and $S_{2}$ (red) in component distance.

The mutual correlative function for the discrete stochastic signals is expressed as follows:

$$
R_{x y}(\tau)=\frac{1}{N} \sum_{n=0}^{N-1} x(n) y(n+\tau) .
$$

Here, $x(n)$ and $y(n)$ are the two signals to be analyzed. $R_{x y}(\tau)$ is the cross-correlation function of the two signals.

Generally, the objects studied in the mutual correlative functions $x(t)$ and $y(t)$ are two different signals; the cross correlative function is neither an even function nor an odd function. As shown in Figure 6, the curves $S_{1}$ and $S_{2}$ are the two discrete stochastic signals. Therefore, $S_{1}$ and $S_{2}$ can be defined as $x(t)$ and $y(t)$, respectively, and the method of cross correlative function can be used to localize the corresponding position of the referential inertial point (the detected inertial point of the curve for the distance in the first cycle) to eliminate the errors.

As shown in Figure 7, when $R_{x y}$ is the largest one, the correlation between $S_{1}$ and $S_{2}$ is ideal; in other words, the two curves are most close. Consequently, $R_{x y}(19,1096)$ in Figure 7 is the maximal, and the cycle ending point can be achieved with $S_{2}$ being moved with detection points $(19,1096)$ along the time domain. The comparison between $S_{1}$ and $S_{2}$ in component distance is shown in Figure 8.

This method was applied to the matching zeros of the curve for the distance of the detected cross sections shown in Figure 3. Then, a spatial spectroscopy scanning can be

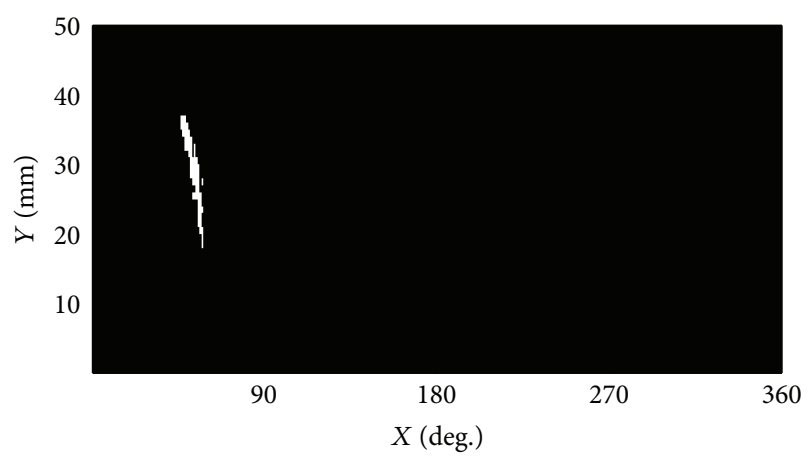

FIGURE 9: Spatial spectroscopy scanning of the component with internal defects.

acquired, as shown in Figure 9. Consequently, the internal defects were detected within much shorter time.

\section{Discussion and Conclusions}

In order to solve the problem of low efficiency in the process of the scanning on the component with complex configuration, we proposed in this paper an approach of matching zeros to detect cross sections, through using the fixed cycle caused by the ellipticity of the component in the process of the scanning with constant speed. Then, a noise reduction method is applied to the periodic signals. Additionally, the method of mutual correlation is adopted to adjust the whole scanning detected without zero sensors. Experimental results show that the detection efficiency of this method is improved by $35 \%$ compared with that of the conventional approach with zero-position sensor. The approach can be expanded to other physical methods for noninvasive detection of internal defect, such as optical spectroscopy or X-ray scanning, and for hybrid medium, such as biological tissues.

This approach has been utilized to detect the defects in components with various shapes. To emphasize the approach rather than the applications, this paper only presents a representative component. The results from other components are similar to the representative one.

The ultrasound backscatter signals are more easily distinguished on the coarse surface than the smooth one when the component is scanned; thus the proposed method is limited for use to detect defects in the components with smooth surface. More advanced methods of pattern recognition will be adopted in the future for improvement of the proposed spatial spectroscopy approach.

To conclude, we proposed a combination of self-adaptive filer and spatial spectroscopy in this study, so as to speed up the scanning component with large change in the surface shape. With this combination, we realized fast detection of internal defect in components.

\section{Competing Interests}

The authors declare that they have no competing interests. 


\section{References}

[1] L. Qi, Z. Wang, Y. Jin, and Z. Ding, "The study of ultrasonic testing method on claviform feedstock of rocket shell body," Journal of Projectiles, Rockets, Missiles and Guidance, vol. 29, no. 4, pp. 269-272, 2009.

[2] B. Xu, X. Yi et al., "Self-adaptive learning based immune algorithm," Journal of Central South University, vol. 19, no. 4, pp. 1021-1031, 2012.

[3] X. Chen, H. Hu, J. Zhang, and Q. Zhou, "An ECT system based on improved RBF network and adaptive wavelet image enhancement for solid/gas two-phase flow," Chinese Journal of Chemical Engineering, vol. 20, no. 2, pp. 359-367, 2012.

[4] H. Feng and Z. Lin, "Design of adaptive sigma-delta A/D converter," High Technology Letters, vol. 11, no. 4, pp. 367-370, 2005.

[5] J. Zhong, Y.-J. Shen, M.-J. Zhao, and L.-Y. Li, "Design of acquisition algorithm based on delay-finding detector for indoor GNSS signals," Journal of Harbin Institute of Technology, vol. 19, no. 5, pp. 6-16, 2012.

[6] X. Ge, D. Luo, and Y. Cao, "Application of correlation function in digial signal processing," Electronics Optics \& Control, vol. 13, no. 6, pp. 78-80, 2006.

[7] L. Ji and H. Zhang, "Study on signal detections based on cross-correlation theory," Journal of Jilin Teachers Institute of Engineering and Technology, no. 6, pp. 39-41, 2004.

[8] Y.-S. Wang and Q.-Q. Ruan, "Application of image orientation and matching algorithm based on correlative matching method," Journal of Northern Jiaotong University, vol. 26, no. 2, pp. 20-24, 2002.

[9] J. Lei, Q. Yao, Y. Lei, and Z. Liu, "Structural damage detection method based on correlation function analysis of vibration measurement data," Journal of Vibration and Shock, vol. 30, no. 8, pp. 221-224, 2011.

[10] A. A. Cavalini, F. S. Lobato, E. H. Koroishi, and V. Steffen, "Model updating of a rotating machine using the self-adaptive differential evolution algorithm," Inverse Problems in Science and Engineering, vol. 24, no. 3, pp. 504-523, 2015.

[11] S. Ghosh, N. Senroy, S. Mishra, and S. Kamalasadan, "Fast power system stabilizer tuning in large power systems," in Proceedings of the IEEE Power \& Energy Society General Meeting, pp. 1-5, Denver, Colo, USA, July 2015.

[12] S. K. Podilchak, J. Shaker, R. Chaharmir, and Y. Antar, "Microwave waveguide transitions using planar anisotropic image guides," in Proceedings of the International Conference on Electromagnetics in Advanced Applications (ICEAA '15), pp. 1416-1418, IEEE, Torino, Italy, September 2015.

[13] N. Bai and B. Lü, "A $200 \mathrm{mV}$ low leakage current subthreshold SRAM bitcell in a $130 \mathrm{~nm}$ CMOS process," Journal of Semiconductors, vol. 33, no. 6, Article ID 065008, pp. 95-100, 2012.

[14] J. Zhong, Y.-J. Shen, M.-J. Zhao, and L.-Y. Li, "Design of acquisition algorithm based on delay-finding detector for indoor GNSS signals," Journal of Harbin Institute of Technology, vol. 19, no. 5, pp. 7-16, 2012.

[15] Y. Deng, Study of threshold de-noising algorithm of speech signal based on wavelet transformation [M.S. dissertation], Chongqing University, 2009.

[16] J. Zhou, J. Wang, H. Yan, S. Li, and G. Gui, "Multiple-response optimization for melting process of aluminum melting furnace based on response surface methodology with desirability function," Journal of Central South University, vol. 19, no. 10, pp. 2875-2885, 2012.
[17] H. Azizpour, R. Sotudeh-Gharebagh, R. Zarghami, and N. Mostouf, "Vibration time series analysis of bubbling and turbulent fluidization," Particuology, vol. 10, no. 3, pp. 292-297, 2012.

[18] G.-G. Meng, T. Takemoto, and H. Nishikawa, "Correlations between IMC thickness and three factors in $\mathrm{Sn}-3 \mathrm{Ag}-0.5 \mathrm{Cu}$ alloy system," Transactions of Nonferrous Metals Society of China, vol. 17, no. 4, pp. 686-690, 2007.

[19] X. Li, J. Song, H. Yuan et al., "Forms and functions of inorganic carbon in the Jiaozhou Bay sediments," Acta Oceanologica Sinica, vol. 28, no. 6, pp. 30-41, 2009. 

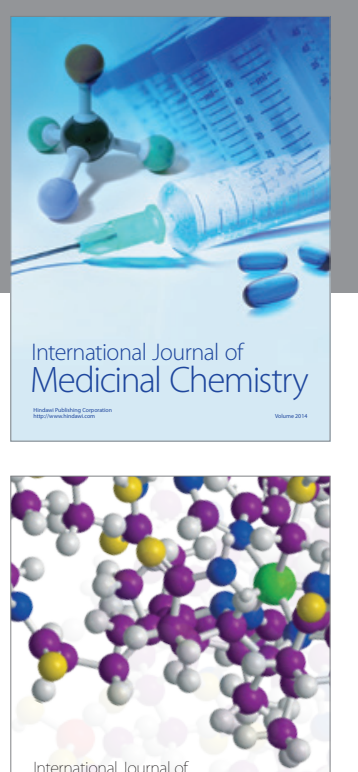

Carbohydrate Chemistry

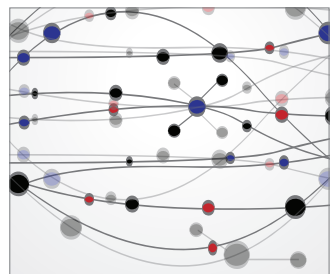

The Scientific World Journal
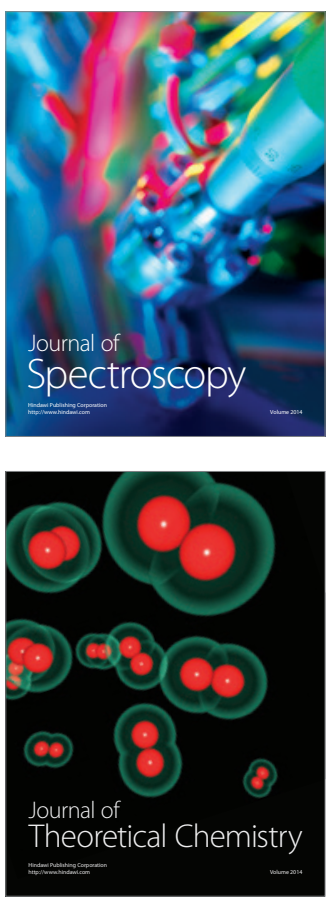
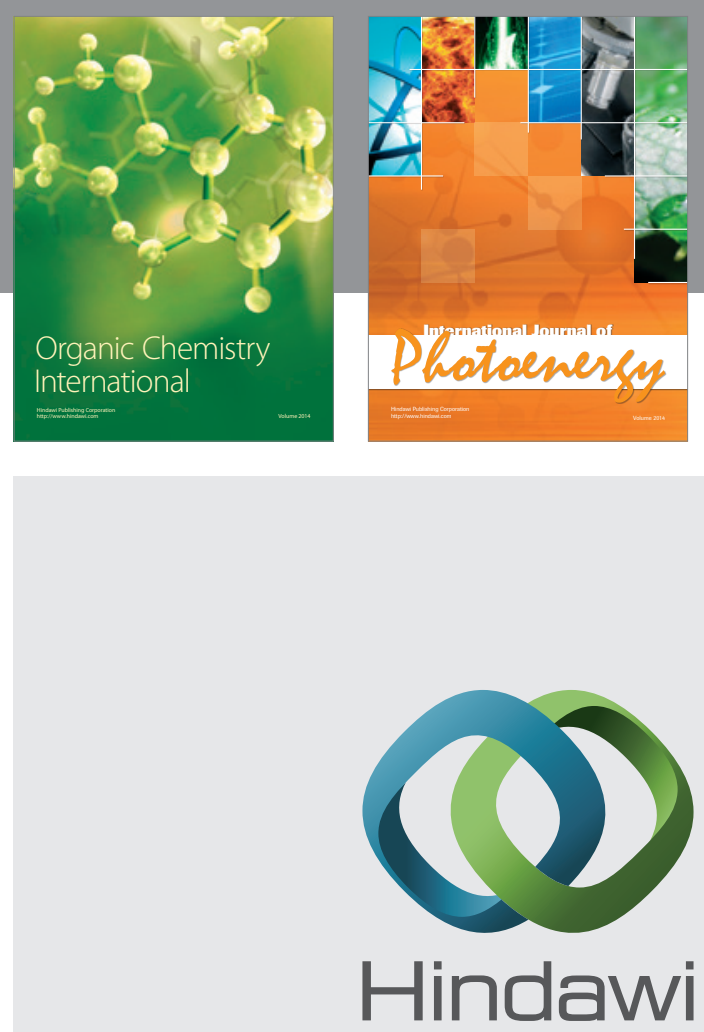

Submit your manuscripts at

http://www.hindawi.com

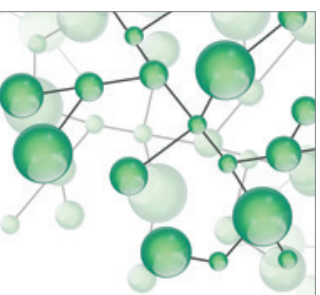

International Journal of

Inorganic Chemistry

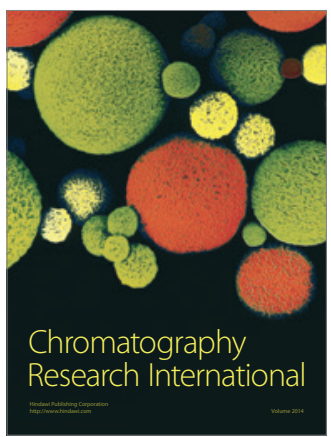

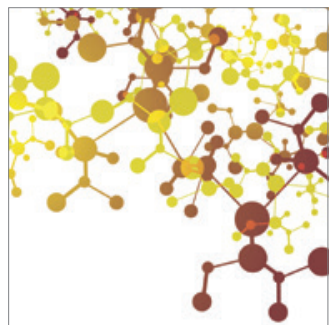

Applied Chemistry
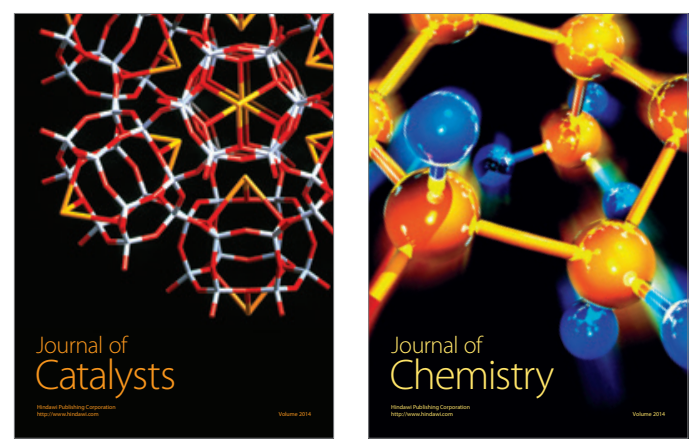
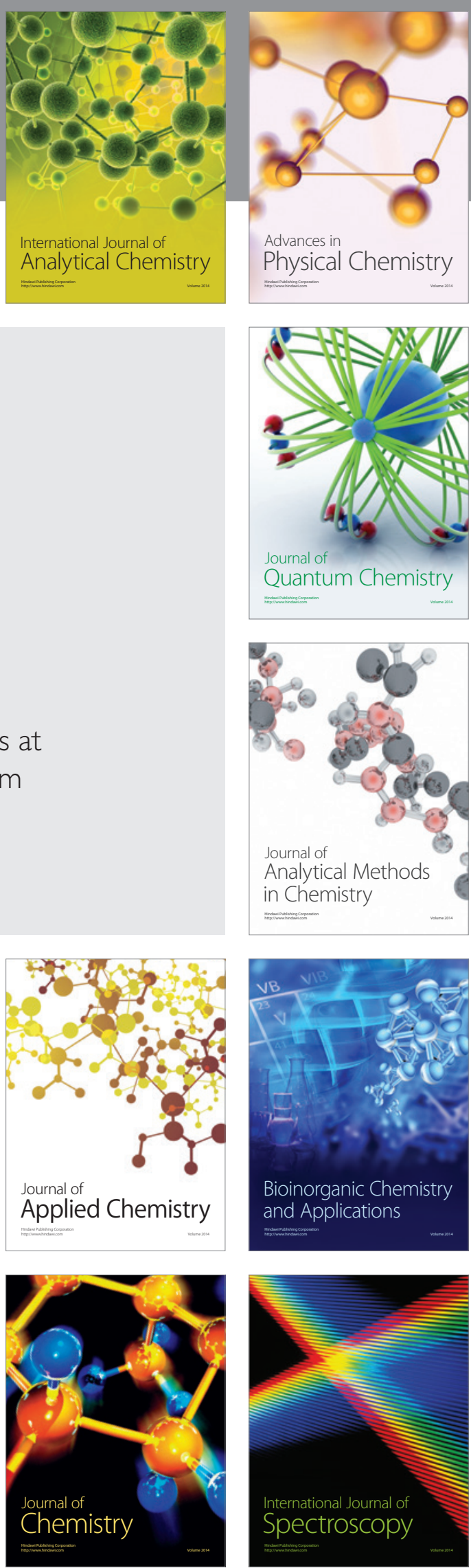